Voix et Images

\title{
Bibliographie de Germaine Guèvremont
}

\section{Joël Boilard, Marie-Ève Landry et Sara-Lise Rochon}

Volume 33, numéro 3 (99), printemps-été 2008

Germaine Guèvremont. Nouvelles survenances

URI : https://id.erudit.org/iderudit/018674ar

DOI : https://doi.org/10.7202/018674ar

Aller au sommaire du numéro

Éditeur(s)

Université du Québec à Montréal

ISSN

0318-9201 (imprimé)

1705-933X (numérique)

Découvrir la revue

Citer ce document

Boilard, J., Landry, M.-È. \& Rochon, S.-L. (2008). Bibliographie de Germaine Guèvremont. Voix et Images, 33(3), 81-93. https://doi.org/10.7202/018674ar d'utilisation que vous pouvez consulter en ligne.

https://apropos.erudit.org/fr/usagers/politique-dutilisation/ 


\title{
B I B L I O GR A P H IE DE GERMAINE G UÈ VREMONT
}

\author{
$+++$ \\ JOËL BOILARD ET MARIE-ĖVE LANDRY \\ Université de Moncton \\ SARA-LISE ROCHON \\ Université d'Ottawa
}

\section{EUVRES DE GERMA INE GUÈ VREM O N T}

I. 1. Livres

I.1.1. En pleine terre

I . 1.1.1. É ditions

+ En pleine terre. Paysanneries. Trois contes, Montréal, Éditions Paysana, 1942, 159 p.

+ En pleine terre, Montréal, Éditions Paysana, 1946, 156 p., illustrations de Cécile Chabot (addition d'un quatrième conte: «Le petit bac du père Drapeau »).

+ En pleine terre, Montréal/Paris, Fides, coll. «Rêve et Vie», 1955, 125 p., gravures sur linoléum de Maurice Petitdidier.

+ En pleine terre, Montréal, Fides, coll. «Goéland», 1976, 140 p., illustrations d'André Bergeron.

+ En pleine terre, Montréal, Bibliothèque Québécoise, 2005, 144 p.

\section{I.1.1.2. Fragments publiés dans des revues}

+ "Les survenants», Paysana, vol. I, n 1, mars 1938, p. 11-12. Repris dans En pleine terre, sous le titre «Chauffe, le poêle ${ }^{1}$ !» $(\mathrm{P})$.

+ «Le départ», Paysana, vol. I, nº 2, avril 1938, p. 12, 32; EPT, «La glace marche» (P).

+ «Sa prière», Paysana, vol. I, n 3, mai 1938, p. 22; EPT, «Prière» (P).

+ «La noce», Paysana, vol. I, no 4, juin 1938, p. 19, 32; EPT, «Une grosse noce» (P).

+ «Un malheur», Paysana, vol. I, no 5, juillet 1938, p. 13; Le Canada, 13 août 1938, p. 2 ; EPT (P).

+ "Quand l'été s'en va...», Paysana, vol. I, n 6, août 1938, p. 11; EPT, «Vers l'automne» (P).

+ «La fille à De-Froi», Paysana, vol. I, no 11, janvier 1939, p. 10-11; EPT, «L'Ange à De-Froi» (P).

+ «Ode à son cheval», Paysana, vol. II, n 1, mars 1939, p. 25; EPT, «Accord» (P).

+ «Un petit Noël», Paysana, vol. II, nº 10, décembre 1939, p. 3 ; EPT (P).

+ «Un vrai taupin», Paysana, vol. IV, n 1, mars 1941, p. 7, 20; EPT (C).

+ «Les demoiselles Mondor», La Revue moderne, vol. XXII, n 1, mai 1941, p. 9, 36; EPT (C). Amérique française, vol. VII, nº 4, juin-août 1949, p. 23-29.

1 Désormais, les références à En pleine terre seront indiqué par le sigle EPT suivi, s'il y a lieu, entre guillemets, du titre sous lequel est présenté le texte. Les lettres P (Paysanneries) et C (Contes) indiquent la section d'En pleine terre dans laquelle le texte est ensuite paru. 
+ «Le bouleau d'argent», Paysana, vol. IV, nº 8, octobre 1941, p. 5, 17; EPT (C).

+ «Un bon quêteux", Paysana, vol. V, nº 7, septembre 1942, p. 4; EPT (P).

+ "Le petit bac du père Drapeau », Paysana, vol. VI, no 8, octobre 1943, p. 5-6; EPT, 2e édition (C).

+ «Vers l'automne», Paysana, vol. XI, nº 8, octobre 1948, p. 9, 21, EPT (P).

\section{I.1.1.3. Paysannerie inédite}

+ «Lettre de Marie Amanda», Bibliothèque et Archives Canada² (LMS 0260, série 1), 4 p. Adaptation monologuée de la nouvelle «Les survenants».

\section{I.1.2. Le Survenant}

I . 1.2.1. É ditions

+ Le Survenant, Montréal, Éditions Beauchemin, 1945, 262 p.

+ Le Survenant, Paris, Librairie Plon, coll. «L'Épi», 1946, 244 p.

+ Le Survenant, Paris, Librairie Plon, 1948, 246 p.

+ Le Survenant, Montréal/Paris, Fides, coll. «Nénuphar», 1959, 198 p., illustrations de Gabriel de Beney.

+ Le Survenant, Montréal/Paris, Fides, coll. «Alouette bleue», 1962, 286 p.

+ Le Survenant, précédé d'une chronologie, d'une bibliographie et de jugements critiques, Montréal/Paris, Fides, coll. «Bibliothèque canadienne-française», 1966, 248 p.

+ Le Survenant, version définitive, Montréal, Fides, coll. "Nénuphar», 1974, 213 p.

+ Le Survenant, édité par Anthony S. Mollica et Gilles P. Deslauriers, Toronto, Copp Clark Publishing Company, 1969, XVI, 283 p. Reproduction phototypique de l'édition parue chez Fides en 1959, sans les illustrations de Gabriel de Beney.

+ Le Survenant, chronologie, bibliographie et jugements critiques d'Aurélien Boivin, Montréal, Fides, coll. «Bibliothèque québécoise», 1974, 233 p.

+ Le Survenant, Montréal, Fides, 1977, 27 f., 52 cm, dix lithographies en couleurs d'André Bergeron. Édition de luxe tirée à 175 exemplaires; texte de la version définitive.

+ Le Survenant, édition critique par Yvan G. Lepage, Montréal, Presses de l'Université de Montréal, coll. «Bibliothèque du Nouveau Monde», 1989, 367 p.

+ Le Survenant, introduction d'Yvan G. Lepage, Montréal, Bibliothèque Québécoise, 1990, 223 p. (nombreux tirages à partir de 1992).

+ Le Survenant, introduction d'Yvan G. Lepage, Montréal, Fides, 2004, 238 p., illustrations de Normand Cousineau.

\section{I . 1 . 2.2. Dact y log raphie}

+ Le Survenant [1942-1944], édition "pré-originale», Bibliothèque et Archives nationales du Québec ${ }^{3}$, Fonds Alfred DesRochers, P6, Centre d'archives de l'Estrie, 209 f.

\section{I.1.2.3. Fragments publiés dans des revues}

+ «L'arrivée de Venant» et "L'abandon», Gants du ciel, nº 2, décembre 1943, p. 21-32 (première version des chapitres I et XVII du Survenant).

\section{$+++$}

2 Désormais: BAC. 3 Désormais: BAnQ. 
+ "Quand le Survenant arrive..., chez les Beauchemin au Chenal du Moine», Paysana, vol. VIII, $\mathrm{n}^{\circ}$ 7, septembre 1945, p. 8 (chapitre I du Survenant; variantes mineures).

\section{I.1.2.4. Traductions (Le Survenant et Marie-Didace en un se ul volume)}

I.1.2.4.1. Traduction anglaise

+ Monk's Reach, trad. par Eric Sutton, Londres, Evans Brothers, 1950, 320 p.

\section{I.1.2.4.2. Traductions a méricaines}

+ The Outlander, trad. par Eric Sutton, New York, Londres/Toronto, Whittlesey House, McGrawHill Book Company, 1950, 290 p.

+ The Outlander, trad. par Eric Sutton, avec une introduction d'Anthony Mollica, Toronto, McClelland and Stewart Limited, coll. «New Canadian Library», n 151, 1978, xv, 290 p.

\section{I.1.3. Marie-Didace}

\section{1.3.1. Éditions}

+ Marie-Didace, Montréal, Beauchemin, 1947, 282 p. (premier tirage: 15 août 1947; second tirage: 15 décembre 1947).

+ Marie-Didace, $2^{\mathrm{e}}$ éd. revue et corrigée, Montréal, Beauchemin, 1948, 282 p. (second tirage: 1953).

+ Marie-Didace, Paris, Librairie Plon, 1949, 239 p. (édition précédée d'une brève analyse du Survenant et suivie d'un glossaire).

+ Marie-Didace, Montréal/Paris, Fides, coll. «Nénuphar», 1956, 210 p., linogravures de Maurice Petitdidier.

+ Marie-Didace, Montréal/Paris, Fides, coll. «Nénuphar», 1958, 210 p., linogravures de Maurice Petitdidier. Réimpression avec corrections de l'édition précédente; deuxième tirage : 28 mai 1965; autres tirages: 1968, 1969, 1971, 1974 et 1976.

+ Marie-Didace, chronologie, bibliographie et jugements critiques d'Aurélien Boivin, Montréal, Fides, coll. «Bibliothèque québécoise», 1980, 229 p. (reproduction en format réduit du tirage de 1976 de l'édition précédente).

+ Marie-Didace, présentation de Jean-Pierre Duquette, Montréal, Bibliothèque Québécoise, coll. «Littérature», 1992, 235 p.

+ Marie-Didace, édition critique par Yvan G. Lepage, Montréal, Presses de l'Université de Montréal, coll. «Bibliothèque du Nouveau Monde», 1996, 446 p.

+ Marie-Didace, présentation de Madeleine Ferron, Montréal, Bibliothèque Québécoise, 2005, $231 \mathrm{p}$.

\section{I . 1.3.2. Dact y log raphie}

+ «Marie-Didace», [s.d.], BAnQ, Fonds Alfred DesRochers, P6, Centre d'archives de l’Estrie, 195 f.

\section{1.3.3. Fragments publiés dans des revues}

+ «Une dernière passée», Gants du ciel, no 8, juin 1945, p. 41-46 (première version du chapitre III de la deuxième partie de Marie-Didace).

+ «Tout s'en va avec le temps», Liaison, vol. I, nº 1, janvier 1947, p. 3-8 (première version du chapitre VIII de la première partie de Marie-Didace). 
+ Marie-Didace. Scènes de la vie canadienne, Le Monde français, vol. VIII, n² 26, novembre 1947, p. 179-212 (1 ${ }^{\text {re }}$ partie, chap. 1-7).

+ Marie-Didace. Scènes de la vie canadienne, Le Monde français, vol. VIII, no 27, décembre 1947, p. 391-417 (1 $1^{\text {re }}$ partie, chap. 8-10).

+ Marie-Didace. Scènes de la vie canadienne, Le Monde français, vol. IX, nº 28, janvier 1948, p. 34-65 (1 ${ }^{\text {re }}$ partie, chap. 11, 12 [12, 13 et 14], 13 [154]).

+ Marie-Didace. Scènes de la vie canadienne, Le Monde français, vol. IX, no 29, février 1948, p. 244-270 (2e partie, chap. 1-3).

+ Marie-Didace. Scènes de la vie canadienne, Le Monde français, vol. IX, no 30, mars 1948, p. 454473 (2 $2^{\mathrm{e}}$ partie, chap. 4-6).

\section{2. Euvres radiophoniques}

\section{I.2.1. Radiorom an s}

\section{I.2.1.1. Le Survenant}

+ Le Survenant, diffusé à CBF, du 10 novembre 1952 au 6 mai 1955, quotidien, 15 minutes, réalisation: Paul Leduc, BAC (LMS 0269, série 1).

+ Le Survenant, CKVL, septembre 1962 - juin 1965, réalisation: A. Cloutier, Archives CKVL, BanQ, MSS-185 et BAC, LMS 0260, série 1 :

+ Première série: épisodes nos 11, 12, 14, 15, 16, 19, 21, 22, 23, 24, 28, 30, 31, 33 et 34 [195 ?]; épisode no 86, 9 mars 1953; épisodes nos 52 à 63, 4 décembre 1962 - 19 décembre 1962 ; épisodes nos 250 à 318, 20 janvier 1964 - 24 avril 1964; épisodes nos 461 à 580, 15 novembre 1954 - 29 avril 1955.

+ Deuxième série (24 septembre 1962 - 31 décembre 1962) : 70 épisodes (les épisodes nos 39 à 68 sont des reprises des épisodes de la série de 1952, transformés, renumérotés et corrigés).

\section{I.2.1.2. Le Déserteur. Les belles histoires des Pays d'en Haut}

+ Le Déserteur. Les belles histoires des Pays d'en Haut, en collaboration avec Claude-Henri Grignon, CBF, 30 septembre 1938 - 29 avril 1939, hebdomadaire, 15 minutes; 30 septembre 1938 9 décembre 1938, tri-hebdomadaire, 15 minutes; 12 décembre 1938 - 29 avril 1939, réalisation: Guy Mauffette, Fonds Archives Radiophoniques et Littéraires (ARL.M.0025.A1), Centre de Documentation Desjardins en études québécoises, Université du Québec à Trois-Rivières, 460 p.

\section{I.2.2. Autres adaptations radiophoniques}

+ La veille d'un Noël paysan, [194 ?], texte d'un radiothéâtre mettant en scène des personnages du Chenal du Moine, BAC (LMS 0260, série 1, 11 p.).

+ Le bouleau d'argent, radio-théâtre, CBF, série «Les voix du pays», 5 octobre 1945, réalisation: Judith Jasmin, BAC (LMS 0260, série 1, 12 p.).

+ Le conte du lièvre, des deux glaçons et des trois boules de neige, $\mathrm{CBF}$, deuxième série des "Contes des enfants sages», 28 décembre 1951, réalisation: Guy Beaulne, 15 minutes, BAC (LMS 0260, série 1, 9 p.).

+ Les demoiselles Mondor, CBF, série "Contes de mon pays», 25 juillet (ou 29 juin) 1954, BAC (LMS 0260, série 1, 43 p.).

4 Les chiffres entre crochets indiquent les chapitres du roman auxquels correspondent les chapitres de la revue. 
+ Les étrennes du curé Labelle, adaptation d'un conte de Joseph Jérôme Grignon, CBF, série "Les Voix du pays», 30 décembre 1945, réalisation: Joseph Beauregard, 30 minutes, BAC (LMS 0260, série 1), 12 p. Repris sous le titre Je me souviens [CBF 5 ?], 4 février 1943, réalisation: Paul Leduc, BAC (LMS 0260, série 1, 9 p.).

+ Le Survenant, adaptation radiophonique du roman, CBF, série «Les Grands romans canadiens», 15 juillet 1951, réalisation: Guy Beaulne, Archives Radio-Canada, 36 p.

+ Marie-Didace, adaptation radiophonique du roman, CBF, série «Les Grands romans canadiens", 22 juillet 1951, réalisation: Guy Beaulne, BAC (LMS 0260, série 1, 32 p.).

\section{3. Euvres télévisuelles}

\section{3. 1. Télé ro m a n s}

\section{I.3.1.1. Le Survenant}

+ Le Survenant, CBFT, 30 novembre 1954 - 9 juillet 1957, réalisation: Maurice Leroux, Fonds de l'Institut de recherches sur la littérature radiophonique et télévisuelle, BAnQ (1 ${ }^{\text {er }}$ mai 1956 9 juillet 1957) et BAC (LMS 0260, série 2):

Première saison : 2 novembre 1954 - 26 avril 1955, 22 épisodes.

Deuxième saison: 18 octobre 1955 - 10 juillet 1956, 21 épisodes.

Troisième saison : 16 octobre 1956 - 9 juillet 1957, 39 épisodes.

Quatrième saison: $1^{\text {er }}$ octobre 1959 - 23 juin 1960, 38 épisodes, Archives Radio-Canada et BAC (LMS 0260, série 2, réalisation: Denys Gagnon.

\section{I.3.1.2. Au Chenal du Moine}

+ Au Chenal du Moine, Radio-Canada, 17 octobre 1957 - 9 juillet 1958, réalisation: Jo Martin, 17 octobre 1957 - 10 juillet 1958, 39 épisodes, BAC (LMS 0260, série 2).

\section{I.3.1.3. Marie-Didace}

+ Marie-Didace, Radio-Canada, 25 septembre 1958 - 26 juin 1959, réalisation: Jo Martin, 17 octobre - 10 juillet 1958, 30 épisodes, Archives Radio-Canada (incomplet: $1^{\text {er janvier } 1959}$ - 26 juin 1959) et BAC (LMS 0260, série 2).

\section{I . 3 . 2. Té lé t h éât r e s}

+ Une grosse nouvelle, CBFT, série «Thêâtre d'été», 23 juin 1954, réalisation: Jean Léonard, 30 minutes, BAC (LMS 0260, série 2, 9 p.).

+ Doré sur tranche, adaptation du conte Les demoiselles Mondor rédigée par Yves Thériault, CBFT, 7-13 août 1955, réalisation: Louis-Georges Carrier, BAC (LMS 0260, série 2), 12 p. Voir La Semaine à Radio-Canada, 7-13 août 1955, p. 7.

\section{3.3. Scénarios de fil ms}

+ L'artisan au pays de Québec, synopsis d'un documentaire sur la valeur de la condition artisanale, [195?], BAC (LMS 0260, série 2), 14 p.

+ L'adieu aux îles, CBFT, série "Provinces», 4 juin et 9 septembre 1968, réalisation: Jean-Paul Fugère, BAC (LMS 0260, série 2).

5 Lieu de rediffusion incertain. 


\section{I.4. Feuilleton Tu seras journaliste ${ }^{6}$}

(Dix-huit épisodes publiés dans Paysana)

+ Paysana, vol. II : no 2, avril 1939, p. 12-13; no 3, mai 1939, p. 28-29; no 4, juin 1939, p. 13, 20 ; no 5, juillet 1939, p. 7, $11 ; n^{\circ}$ 6, août 1939, p. 10-11; nº 7, septembre 1939, p. 8-9; no 8, octobre 1939, p. 6-7; nº 9, novembre 1939, p. 6, 7, 13; no 11, janvier 1940, p. 5-7; nº 12, février 1940, p. 6-7.

+ Paysana, vol. III : $n^{\circ}$ 1, mars 1940, p. 18-19; nº 1bis, avril 1940, p. 22-23; nº 2, mai 1940, p. 24, 25, 32; no 3, juin 1940, p. 20-22; no 4, juillet 1940, p. 9, 24; no 5, août 1940, p. 19, 24 ; $\mathrm{n}^{\circ} 6$, septembre 1940, p. 20-21; n 7 , octobre 1940, p. 22-24.

\section{5. Nouvelles}

+ "Le rêve d'un chef ", Paysana, vol. I, n 8, octobre 1938, p. 19.

+ «Une grosse nouvelle», Contes de l'Almanach (1938?), BAC (LMS 0260, série 3, 20 p.).

+ «Un sauvage ne rit pas», La Revue moderne, vol. XXIV, n 11, mars 1943, p. 10-11.

+ «Lettre morte», Paysana, vol. VI, no 3, mai 1943, p. 8-9.

+ "La peur», Paysana, vol. VI, nos 5-6, juillet-août 1943, p. 14.

+ "Le chambreur (conte drolatique inscrit au concours littéraire)", Amérique française, vol. III, $\mathrm{n}^{\circ}$ 5, septembre-octobre, 1951, p. 12-17.

+ «L'émeraude, nouvelle sentimentale», La Revue moderne, vol. XXXIV, nº 1, mai 1952, p. 8, 17, 21.

+ "Au pays du Survenant», La Revue moderne, vol. XXXIX, n 1, mai 1957, p. 12, 14.

+ «Le plomb dans l'aile», Cahiers de l'Académie canadienne-française, vol. IV, 1959, p. 69-75.

\section{I.6. Chroniques}

\section{I.6.1. Sous le nom "Germaine Guèvremont"}

+ «Faites votre possible. Il n'y a pas de recette de roman », Le Petit Journal, 30 septembre 1951, p. 55.

\section{I.6.1.2. Chroniques publiées dans Le Nouveau Journal}

+ «Un homme habité», 9 septembre 1961, p. 21 ( $\left(3\right.$ minutes avec ${ }^{7} »$ ).

+ «Le chemin des roses», 16 septembre 1961, p. 18.

+ «Cocktail et fantaisie», 23 septembre 1961, p. 20.

+ «Anatomie d'un faire-part», 30 septembre 1961, p. 20.

+ «La maison de ma tante [Alzire Grignon-Lachaîne] », 7 octobre 1961, p. 23.

+ «Sur trois échos», 14 octobre 1961, p. 20.

+ «Ma vie est un roman», 21 octobre 1961, p. 22.

+ «Aimez-vous... Schopenhauer?», 28 octobre 1961, p. 19.

+ «Découpages», 4 novembre 1961, p. 20.

+ «Onze novembre», 11 novembre 1961, p. 22.

+ «Ne me donnez, Seigneur...», 18 novembre 1961, p. 21.

+ «Le premier scoop du monde», 25 novembre 1961, p. 19.

6 Tu seras journaliste a été publié sous forme de feuilleton seulement, jamais dans son intégralité. $\quad 7$ Tous les textes publiés dans Le Nouveau Journal figurent dans la chronique « 3 minutes avec». 
+ «Où allons-nous?», 2 décembre 1961, p. 22.

+ «Ce vice puni... la lecture», 9 décembre 1961, p. 23.

+ «Information-minute», 16 décembre 1961, p. 20.

+ «Noël approche», 23 décembre 1961, p. 22.

+ «Les visiteurs du jour de l'an», 30 décembre 1961, p. 21.

+ «D'une étrenne à l'autre», 6 janvier 1962, p. 21.

+ «Sur un air connu», 13 janvier 1962, p. 23.

+ «Bon à tirer», 20 janvier 1962, p. 21.

+ «Nouvelle révolution», 27 janvier 1962, p. 23.

+ «Voyage au pays de la bonne fourchette», 3 février 1962, p. 23.

+ «La dame errante», 10 février 1962, p. 21.

+ «Un coup de fil», 17 février 1962, p. 21.

+ «À la croque au sel», 24 février 1962, p. 23.

+ «Enfants de la nuit», 3 mars 1962, p. 20.

+ «Connaissez-vous Annie?», 10 mars 1962, p. 25.

+ «Deux nouvelles», 17 mars 1962, p. 23.

\section{I.6.2. Sous le pseudonyme "Janrhêve ${ }^{8}$ "}

+ «Trouvé: un journal», L'Étudiant, vol. III, nº 12, 6 février 1914, p. 3.

+ «Un cœur passa...», L'Étudiant, vol. III, no 14, 20 février 1914, p. 3.

+ [Sans titre], L'Étudiant, vol. III, nº 15, 27 février 1914, p. 4.

+ «Lettre au grand-père», L'ÉEtudiant, vol. III, no 17, 13 mars 1914, p. 4.

+ [Sans titre], L'Étudiant, vol. III, nº 18, 20 mars 1914, p. 4.

+ [Sans titre], L'Étudiant, vol. III, no 19, 27 mars 1914, p. 4.

+ «Un conte», L'Étudiant, vol. III, nº 20, 2 avril 1914, p. 6.

+ «Le monde où l'on s'amuse. Aux sucres», L'Étudiant, vol. III, nº 22, 24 avril 1914, p. 6.

+ «La jeunesse», L'Étudiant, vol. III, no 23, 1 ${ }^{\text {er }}$ mai 1914, p. 6 («Le monde féminin »).

+ «La garde», La Patrie, 17 octobre 1914, p. 15 («Le royaume des femmes»).

+ «Novembre», La Patrie, 28 novembre 1914, p. 19 («Le royaume des femmes»).

+ [Sans titre], L'Étudiant, vol. IV, n 1, 4 décembre 1914, p. 4 («Chronique féminine»).

+ [Sans titre], Le Canada, 11 octobre 1917, p. 9 («Coin des étudiants»).

+ «Ā quel âge?», L'Avenir du Nord, 1 ${ }^{\text {er }}$ septembre 1933, p. 2.

+ «Propos littéraires. Un homme et son péché», Le Droit, 3 mars 1934, p. 4.

+ «Écrivons-nous. La semaine de la lettre», Paysana, vol. II, nº 9, novembre 1939, p. 22.

+ «Madame Rivard», [journal non identifié], [194 ?] (« Des riens en Prose»), BAC (LMS 0260, série 4), 1 p.

\section{I.6.3. Sous le pseudonyme "La Passante" dans la revue Paysana 9}

+ Paysana, vol. I: n 1, mars 1938, p. 28; n², avril 1938, p. 28-29; n 3, mai 1938, p. 28-29; nº 5, juillet 1938, p. 24-25; n 6, août 1938, p. 23-24.

8 Les sept premiers textes ainsi que «La jeunesse» ont été publiées dans le cadre de la chronique "Le monde féminin ». 9 Sous le titre «Pays-Jasettes», avec la collaboration de Françoise Gaudet-Smet. 
+ Paysana, vol. II : no 2, avril 1939, p. 28-29; no 3, mai 1939, p. 14-15; no 4, juin 1939, p. 24-25; $\mathrm{n}^{\circ}$ 5, juillet 1939, p. 14; n 10, décembre 1939, p. 20; n 12, février 1940, p. $24,31$.

+ Paysana, vol. III : n 10, décembre 1940, p. 18

+ Paysana, vol. IV : no 1, mars 1941, p. 6, 24; n 2, avril 1941, p. 2; n 3, mai 1941, p. 5; n 4, juin 1941, p. $14 ; n^{\circ} 7$, septembre 1941, p. $24 ; n^{\circ} 12$, février 1942, p. 21-22.

+ Paysana, vol. V: no 2, avril 1942, p. 2; n 10, décembre 1942, p. 4; no 11, janvier 1943, p. 4.

+ Paysana, vol. VI: $\mathrm{n}^{\circ}$ 1, mars 1943, p. 4.

+ Paysana, «L'arbre devant la maison", n 2, avril 1943, p. 16 (déjà publiée, avec variantes, dans L'CFil, vol. I, no 10, 15 mai 1941, p. 25).

\section{I.6.4. Sous le pseudonyme "La Femme du Postillon"}

+ «La raison d'un pseudo", L'EFil, vol. I, n 5, 15 décembre 1940, p. 27 ("Courrier extraordinaire $\left.{ }^{10} »\right)$.

+ «Un quêteux sans pareil», L'CFil, vol. I, nº 6, janvier 1941, p. 32.

+ "La petite "campe" de l’Enfant Jésus», L'EFil, vol. I, nº 7, 15 février 1941, p. 19.

+ «Soleil», L’EFil, vol. I, nº 8, 15 mars 1941, p. 25.

+ «Propos de printemps», L'EFil, vol. I, no 9, 15 avril 1941, p. 26.

+ "L'arbre devant la maison ", L'CFil, vol. I, n 10, 15 mai 1941, p. 25 (repris, avec variantes, dans Paysana, vol. VI, n² 2, avril 1943, p. 16).

+ "À la manière de... », L'CEil, vol. I, no 11, 15 juin 1941, p. 30.

+ «La gloire d'une débutante», L'EFil, vol. I, nº 12, 15 juillet 1941, p. 32.

+ «Monique en vacances», L'CFil, vol. II, no 1, 15 août 1941, p. 26.

+ «La dame de journée moyen-âge», L'CEil, vol. II, n 2, 15 septembre 1941, p. 24.

+ «Plus précisément...", L'EFil, vol. II, n 3, 15 octobre 1941, p. 21.

+ «Portés disparus», L'CEil, vol. II, n 4, 15 novembre 1941, p. 25.

+ «Thémis versus Diane», L’EFil, vol. II, nº 5, 15 décembre 1941, p. 26.

+ «En marge de la guerre», L'CEil, vol. II, nº 6, 15 janvier 1942, p. 23.

\section{7. É crits journalistiques}

+ «La femme du médecin de campagne», Paysana, vol. I, no 8, octobre 1938, p. 17-18.

+ «Quand ils questionnent», Familia, vol. VI, n³, mars 1939, p. 14-15.

+ «Le 3e centenaire de Racine», La Revue populaire, vol. XXXII, n 11 , novembre 1939, p. 7.

+ «Louis Fréchette», Paysana, vol. II, no 10, décembre 1939, p. 6.

+ «Un ami des livres», La Revue populaire, vol. LXXIII, n 5, mai 1940, p. 9, 62, 67.

+ "La Société des écrivains canadiens», Culture, vol. I, nº 3, septembre 1940, p. 362-363.

+ "C'est notre fête», Paysana, vol. IV, nº 1, mars 1941, p. 24.

+ «Une femme et son métier», Paysana, vol. IV, n 10, décembre 1941, p. 4.

+ «La femme, péril ou salut de la terre», Paysana, vol. IV, n 11, janvier 1942, p. 6 (repris dans Le Monde rural, Montréal, Éditions Almanach-Magazine de la Jeunesse agricole catholique, 1942, p. 87-86).

+ «Paysana présente... Marie-Claire Daveluy», Paysana, vol. IV, nº 12, février 1942, p. 8.

$$
+++
$$

10 Tous les textes publiés dans la revue L'EFil figurent dans la chronique «Courrier extraordinaire». 
+ «Une famille au service de l'agriculture», Paysana, vol. V, nº 1, mars 1942, p. 14-15.

+ «Une belle carrière. Florine Phaneuf», Paysana, vol. V, n 3, mai 1942, p. 9.

+ «Le 3e centenaire de Sorel», Le Samedi, vol. LIV, no 12, 15 août 1942, p. 4, 5, 31.

+ «Nos grandes femmes. Une jeunesse de 83 ans», Paysana, vol. V, n 10, décembre 1942, p. 9.

+ «De fil en aiguille», Paysana, vol. VI, nº 1, mars 1943, p. 7.

+ «Un maître artisan: Moïse Beauchemin, pionnier de l'industrie des machines aratoires dans la province de Québec», Paysana, vol. VI, n² 2, avril 1943, p. 8-9.

+ "L'enfant, notre espoir...», Paysana, vol. VI, no 7, septembre 1943, p. 9.

+ "La découverte de Sorel en 1926 », Paysana, vol. VI, n 9, novembre 1943, p. 6-7.

+ "Figures de femmes. "Il y a soixante ans"», Paysana, vol. VI, n 10, décembre 1943, p. 5.

+ "Figures de femmes. Madeleine Huguenin", Paysana, vol. VI, no 10, décembre 1943, p. 8.

+ «En ce temps-là le premier baptisé canadien», Paysana, vol. VI, n 11, janvier 1944, p. 6, 13.

+ «Fidélité», Paysana, vol. VI, nº 12, février 1944, p. 3.

+ " ... des mains ingénieuses... », Paysana, vol. VI, n 12, février 1944, p. 8.

+ "Avec un grain de sel...", Paysana, vol. VII, nº 1, mars 1944, p. 6, 12.

+ «Un chapeau se penche sur une femme», Paysana, vol. VII, n² 2, avril 1944, p. 7, 18.

+ "L'Académie canadienne-française», La Revue populaire, vol. XXXVIII, nº 2, février 1945, p. 5.

+ «Marguerite Lemieux, fille de France, née au Canada», Paysana, vol. V, nº 1, mars 1945, p. 8-9.

+ «L'Artisanat et nos artisans. Georgette DuPerré», Paysana, vol. VIII, nºs 5-6, juillet-août 1945, p. 8-9.

+ «Goût de la lecture: richesse insurpassable!», Paysana, vol. IX, nº 12, février 1947, p. 4.

+ «La langue paysanne du Canada», Liaison, vol. III, n² 25, mai 1949, p. 274-278.

+ «Marie Mauron», Cahiers de l'Académie canadienne-française, vol. XI, 1967, p. 144-150.

\section{I.8. É crits autobiographiques}

+ «Le tour du village», Paysana, vol. IV, nº 3, mai 1941, p. 6.

+ «Le tour du village», Paysana, vol. IV, nº 4, juin 1941, p. 10.

+ «Le tour du village», Paysana, vol. IV, nos 5-6, juillet-août 1941, p. 10.

+ «Le tour du village», Paysana, vol. VI, no 4, juin 1943, p. 14.

+ "Portrait de mon père, Joseph Jérôme Grignon », Société royale du Canada. Présentation, n 16, 1961-1962 (séance du 14 avril 1962), p. 93-98.

+ «Jamais je n’oublierai... », dans Présence de Victor Barbeau, Montréal, Ateliers Pierre Desmarais, 1963, cahier n² 2, p. 23-26.

+ «À l'eau douce. Extraits des mémoires de Germaine Guèvremont», Châtelaine, vol. VIII, no 4 , avril 1967, p. 34, 35, 74, 76, 78, 80, 82.

+ «Le premier miel. Un chapitre inédit de Germaine Guèvremont», Le Devoir, 31 octobre 1967, p. XXI.

\section{9. Préf a c e s}

+ BEAUDOIN, Juliette, Bio-bibliographie de Vieux Doc, thèse présentée à l'École des bibliothécaires, Montréal, Université de Montréal, 1946, 40 p.

+ GRAY, Clayton, Montréal qui disparaît, Montréal, J-A. Pony, 1952, p. 7.

+ BROCHU, André, J.-André CONTANT et Yves FLUBÉ, Étranges domaines, Montréal, Éditions de la Cascade, 1957, p. 3-4.

+ PELLETIER-DLAMinI, Louis, Pomme-de-Pin, Montréal, Éditions de l'Homme, 1968, p. 5-7. 


\section{10. Conférences in édites}

+ «Les petites joies d'un grand métier», conférence donnée à l'automne [?] 1945, Bibliothèque municipale de Montréal, série «Votre auteur préféré»; BAnQ, Fonds Alfred DesRochers, P6, Centre d'archives de l'Estrie, $19 \mathrm{p}$.

+ [Sans titre], texte d'une conférence sur le métier de journaliste, Toronto, [1949 ?], BAC (LMS 0260, série 5, 26 p.).

+ "Les prix Nobel et les femmes», Société d'étude et de conférences de Montréal, 28 février 1950, BAC (LMS 0260, série 5, 12 p.). Résumé de G. B. dans Le Devoir, $1^{\text {er }}$ mars 1950, p. 5.

+ «Le français qui étonne la France de nos jours», texte d'une causerie, [195 ?], BAC (LMS 0260, série 5, 17 p.).

+ "Le parlé au Canada est un sujet complexe», Association des Humanités, [195 ?], BAC (LMS 0260, série 5, 10 p.).

+ «Débuts comme romancière», texte d'une allocution sur ses débuts de romancière, [195 ?], public non identifié, BAC (LMS 0260, série 5, 3 p.).

+ "À Banff», dossier contenant le texte prononcé par Germaine Guèvremont à l'occasion de la réception du prix du Gouverneur général pour The Outlander et le texte de sa participation à un panel organisé par la Canadian Author's Association, 1951, BAC (LMS 0260, série 5, 21 p.).

+ «How did I start to write?», [1951 ?], texte incomplet, public non identifié, BAC (LMS 0260, série 5,5 p.).

+ «Le Survenant, homme ou dieu?», texte dactylographié, 24 novembre 1965, Archives générales, Centre d'Études canadiennes-françaises, Université de Montréal. Résumé de Robert Barberis dans Le Quartier latin, 2 décembre 1965, p. 9.

\section{II. ÉTUdES CONSACRÉES À GERMAINE GUÈVREMONT 11}

\section{I . 1 . Liv res}

+ BAILLIE, Robert, Le Survenant: lecture d'une passion, Montréal, XYZ éditeur, 1999, 183 p.

+ LACHAPELLE, Jean-Jacques et al., Le Survenant et son temps, Sorel, Centre d'interprétation du patrimoine de Sorel, 1997, $30 \mathrm{p}$.

+ LEPAGE, Yvan G., Germaine Guèvremont. La tentation autobiographique, Ottawa, Presses de l'Université d'Ottawa, coll. «Euvres et auteurs», 1998, 205 p.

+ OLIVIER, Chantal, Le règne de la famille ou Le père Didace dans Le Survenant de Germaine Guèvremont, Joliette, édition privée, coll. «Euvres bibliophiliques de Lanaudière», 1993, 52 p.

\section{II.2. Chapitres ou extraits de livres}

+ CAGNON, Maurice, "Germaine Guèvremont», dans Romanciers canadiens, Québec, Presses de l'Université Laval, coll. «Vie des lettres canadiennes», 1972, p. 47-53.

+ DÉCARIE, David, «Le rêve de Phonsine. L'enchâssement d'un rêve dans le roman Marie-Didace de Germaine Guèvremont», Le récit de rêve, Christian Vandendorpe (dir.), Québec, Nota bene, 2005, p. 317-338.

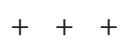

11 Nous avons retenu les livres, études, thèses (et autres manifestations) postérieurs aux éditions critiques du Survenant et de Marie-Didace, présentées par Yvan G. Lepage, auxquelles nous renvoyons le lecteur; nous avons aussi fait l'économie de certains textes qui se limitaient à mentionner au passage Germaine Guèvremont ou son œuvre. 
+ DÉCARIE, David, «Résonances. Interfiguralité chez Germaine Guèvremont», La rhétorique au féminin, Annette Hayward (dir.), Québec, Nota bene, 2006, p. 315-333.

+ GÉLINAS, François, "Germaine Guèvremont 1893-1968», Le Survenant et son temps, JeanJacques Lachapelle et al., Sorel, Centre d'interprétation du patrimoine de Sorel, 1997, p. 9-14.

+ GIROUARD, Pierre, «Le Survenant et la Révolution tranquille», Le Survenant et son temps, Jean-Jacques Lachapelle et al., Sorel, Centre d'interprétation du patrimoine de Sorel, 1997, p. 15-20.

+ JOUBERT, Lucie, "Les voix de Germaine Guèvremont: Tu seras journaliste», La passion des lettres. Études de littérature médiévale et québécoise en hommage à Yvan Lepage, Pierre Berthiaume et Christian Vandendorpe (dir.), Ottawa, Éditions David, coll. «Voix savantes», 2006, p. 215-224.

+ LACHAPELLE, Jean-Jacques, «Mobilité au temps du Survenant», Le Survenant et son temps, JeanJacques Lachapelle et al., Sorel, Centre d'interprétation du patrimoine de Sorel, 1997, p. 7-26.

+ LEFEBVRE, Richard, «Les survenants au Chenal du Moine», Le Survenant et son temps, JeanJacques Lachapelle et al., Sorel, Centre d'interprétation du patrimoine de Sorel, 1997, p. 25-28.

+ LEPAGE, Yvan G., «Guèvremont, Germaine», Encyclopedia of Literature in Canada, Toronto, University of Toronto Press, 2002, p. 458-459.

+ LEPAGE, Yvan G., «Les chansons dans l'œuvre de Germaine Guèvremont», Croire à l'écriture. Études de littérature québécoise en hommage à Jean-Louis Major, Yvan G. Lepage et Robert Major (dir.), Orléans, Éditions David, 2000, p. 205-224.

+ LEPAGE, Yvan G., «Mort et transfiguration du roman de la terre», Le Survenant et son temps, Jean-Jacques Lachapelle et al., Sorel, Centre d'interprétation du patrimoine de Sorel, 1997, p. 21-24.

+ MAJOR, André, Le sourire d'Anton ou l'adieu au roman. Carnets 1975-1992, Montréal, Presses de l'Université de Montréal, 2001, 208 p. (en particulier p. 77-79).

+ VANASSE, André, «Le Survenant, roman de Germaine Guèvremont (née Grignon)», dans Dictionnaire des œuvres littéraires du Québec, t. III, 1940-1959, Montréal, Fides, 1982, p. 953-959.

\section{I . 3. A r ticles}

+ ALLARD, Jacques, "Deux scènes médianes où le discours prend corps», Études françaises, vol. XXXIII, nº 3, hiver 1997, p. 53-65.

+ BLAIS, Suzelle, "Quelques mots tirés du roman Le Survenant», Québec français, n 136, hiver 2005, p. 97-99.

+ BOIVIN, Aurélien, «Le Survenant», Québec français, nº 99, automne 1995, p. 90-94.

+ CAMBRON, Micheline, «La ville, la campagne, le monde: univers référentiels et récit», Études françaises, vol. XXXIII, n 3, hiver 1997, p. 23-35.

+ DÉCARIE, David, «La même turelure. Fête et répétition dans le Cycle du Survenant de Germaine Guèvremont», Port Acadie, nos 8-9, automne 2005-printemps 2006, p. 159-170.

+ DÉCARIE, David, «Le relais des survenants chez Germaine Guèvremont», Voix et Images, vol. XXVI, n² 2, hiver 2001, p. 359-383.

+ FORTIER, Frances, DUPONT, Caroline et Robin SERVANT, «Quand la biographie se "dramatise" : le biographique d'écrivain transposé en texte théâtral", Voix et Images, vol. XXX, $\mathrm{n}^{\circ}$ 2, hiver 2005, p. 79-104.

+ FOURNIER, Isabelle, «Le mythe de la mère et la dénégation de la sexualité féminine dans les romans de la terre au Québec», Québec français, no 137, printemps 2005, p. 47-49, 58. 
+ GAULIN, Michel, "Compte rendu du livre Marie-Didace de Germaine Guèvremont», Lettres québécoises, no 91, automne 1998, p. 44.

+ HUDON, Jean-Guy, "Compte rendu du livre Marie-Didace de Germaine Guèvremont», Nuit blanche, no 71, été 1998, p. 8-12.

+ LAMARRE, Sylvie, Laure NEUVILLE et Lori SAINT-MARTIN, "Sexe, pouvoir et dialogue», Études françaises, vol. XXXIII, no 3, hiver 1997, p. 37-52.

+ LAMONTAGNE, André, "Écrire l'Amérique», Voix et Images, vol. XXIII, n 3, printemps 1998, p. 612-617.

+ LE BRAS, Yvon, "Altérité et identité dans Le Survenant de Germaine Guèvremont", Francographies. Bulletin de la société des professeurs français et francophones d'Amérique, vol. IX, 2000, p. 43-52.

+ LE BRAS, Yvon, «Le Saint-Laurent dans l'œuvre romanesque de Germaine Guèvremont», Études canadiennes/Canadian Studies, vol. L, juin 2001, p. 177-187.

+ LEPAGE, Yvan G., "Germaine Guèvremont et la figure du père», Société royale du Canada. Présentations, vol. LI, Ottawa, Société royale du Canada, Académie des lettres et des sciences humaines, 1998, p. 11-24.

+ MARCOTTE, Gilles, «Restons traditionnels et progressifs, disait Onésime Gagnon », Études françaises, vol. XXXIII, n³, hiver 1997, p. 5-13.

+ MORENCY, Jean, «Deux visions de l'Amérique», Études françaises, vol. XXXIII, n 3, hiver 1997, p. 65-77.

+ NOËL, Denise, «Pure laine: une lecture du Survenant de Germaine Guèvremont», Conjonctures, $\mathrm{n}^{\circ} 18$, printemps 1993, p. 61-77.

+ OLSCAMP, Marcel, «Un pari institutionnel : l'édition critique au Québec», Études françaises, vol. XXVIII, no 1, automne 1992, p. 133-170.

+ SMART, Patricia, "“Changer la vie" ou "changer le monde"? ", Études françaises, vol. XXXIII, no 3, hiver 1997, p. 15-21.

+ TRUDEL, Danielle, «Ces étrangers d'ici et d'ailleurs», Québec français, no 97, printemps 1995, p. 77-80.

\section{I . 4. Mémoires et $t h$ è s e s}

+ BOND, Helen Martha, The Decline of the Family Farm in the Canadian Novel, 1925-1952, mémoire de maîtrise, Université Laval, 1995, 129 f.

+ GAGNÉ, Marie-Pierre, «"Aussi belle fille que Maria Chapdelaine". La littérature dans la revue féminine rurale Paysana (1938-1949)", mémoire de maîtrise, Université Laval, 2007, 121 f. (le chapitre 3 porte sur Germaine Guèvremont, f. 65-97).

+ GILBERT, Danielle, «Le Survenant. Inscription idéologique ou variation sur une nostalgie du terroir ", mémoire de maîtrise, Université du Québec à Montréal, 1984, 154 f.

+ LI, Ping Qing, «L'autre dans Le Survenant de Germaine Guèvremont. Éléments pour une nouvelle lecture», mémoire de maîtrise, Université de Moncton, 1992, 105 f.

+ MARTIN, Stefanie, «La figure du Christ dans l'œuvre romanesque de Germaine Guèvremont», mémoire de maîtrise, Université McGill, 1991, 146 f.

+ MIRATON, Catherine, «Regard de Germaine Guèvremont sur la fin d'une dynastie paysanne. Le Survenant, Marie-Didace, derniers romans de la terre», thèse de maîtrise, Université de Bourgogne, 1993, $73 \mathrm{f}$.

+ POIRIER, Michel Philippe, "Le Survenant selon Giono et Guèvremont», mémoire de maîtrise, Université McGill, 1988, 108 f. 
+ ROBERTS, Katherine Ann, "Le roman national des femmes du Québec (1891-1984)", thèse de doctorat, Université Queen's, 1999, 238 f. (le chapitre 4 porte sur Germaine Guèvremont et Marie Le Franc, f. 95-138).

+ THÉRIAULT, Michel, «Journal sans bord suivi de Rêve et roman», mémoire de maîtrise, Université Laval, 1991, 225 f.

+ THÉRIAULT, Michel, «Vers une méthode d'analyse et d'interprétation du rêve fictif », thèse de doctorat, Université Queen's, 1996, 418 f.

+ THOMAS, Jean-Pierre, «Essai de mythanalyse de l'imaginaire québécois du Xxe siècle (19161945) à partir de textes romanesques représentatifs », thèse de doctorat, Université de Sherbrooke, 2003, $451 \mathrm{f}$.

+ TRUDEL, Danielle, «L'influence du personnage excentrique dans Le Survenant et quelques romans québécois du Xxe siècle», thèse de doctorat, Université Laval, 2002, 225 f.

\section{I I . F I L M}

+ Le Survenant, Les Films Vision 4, 2005, réalisation: Érik Canuel, 138 minutes. Avec JeanNicolas Verrault, Gilles Renaud, François Chénier, Catherine Trudeau, Patrice Robitaille, Anick Lemay. 